\title{
Dielectric properties of the PFN ceramics obtained different chemical-wet technology and sintering by hot pressing method
}

\author{
Dariusz Bochenek ${ }^{1}$ and Przemysław Niemiec ${ }^{1, a}$ \\ ${ }^{1}$ University of Silesia in Katowice, Faculty of Computer Science and Material Science, Institute of Technology and Mechatronics \\ 12, Żytnia St., 41-200, Sosnowiec, Poland
}

\begin{abstract}
The paper shows three kinds of chemical-wet technology synthesis of the ferroelectric-ferrimagnetic $\mathrm{PbFe}_{1 / 2} \mathrm{Nb}_{1 / 2} \mathrm{O}_{3}$ materials and technology process of the ceramic samples sintered by hot pressing method. In the technological process of the PFN powders were used follows precursors: lead acetate trihydrate and niobium ethoxide while an iron-related components were changed: iron nitrate obtained by first sol-gel technology (PFN-n), iron oxalate obtained by second sol-gel technology (PFN-ox) and iron citrate obtained by third technology (PFN-c). XRD, SEM tests and temperature measurements of dielectric properties of the ceramic samples were carried out. The SEM microstructure tests of the PFN ceramic samples confirm the properly conducted technological processes. The best dielectric properties of the PFN ceramic samples were obtained for third technology using iron citrate precursor. But, this method of the PFN ceramic powder requires minimization of a pyrochlore phase and reduction porosity of the ceramic samples.
\end{abstract}

\section{Introduction}

Multiferroics are smart materials which show simultaneously at least two types of physical (ferroic) states e.g. a ferromagnetic state (antiferromagnetic, ferrimagnetic), a ferroelectric state (antiferroelectric, ferrielectric), a ferroelastic state (ferromagneticelastic, ferroelastoelectric), ferrotoroid state and showing coupling between subsystems [1-4]. Ceramic materials with multiferroic properties found functional application as various types of piezoelectric transducers, electric bandfilters, sensors, generators, servomotors, phase modulators, frequency multipliers, electromechanical, electroacoustic, pyroelectric transducers, memory elements, etc. [5-7]. These tape multiferroic materials are designed in the form of solid solutions [8] and multicomponent compounds [9-10], thin films [11], and composites [12-15].

The ferroelectromagnetic $\mathrm{PbFe}_{1 / 2} \mathrm{Nb}_{1 / 2} \mathrm{O}_{3}$ ceramics, shortly called PFN, is one of the materials belonging to group of multiferroics. The PFN ceramics has perovskite structure with the general $\mathrm{A}\left(\mathrm{B}_{1-\mathrm{x}} \mathrm{B}_{\mathrm{x}}\right) \mathrm{O}_{3}$ formula, where $\mathrm{Pb}^{2+}$ in site $\mathrm{A}$ and $\mathrm{Nb}^{5+}$ in site $\mathrm{B}$ favour electrical order and $\mathrm{Fe}^{3+}$ in the $\mathrm{B}$ site gives rise to a magnetic moment that generates magnetic order [16].

Many methods and techniques for obtaining PFN ceramic powders are known e.g. a molten salt synthesis, a reaction in solid state, two-stage columbite, sol-gel, coprecipitation method, wolframite route, high-energy milling and others [17-20]. Each of these methods requires a used of specific technological conditions and diligence in laboratory process in order to obtained materials with good properties.

The aim of the work was obtained and studied the ferroelectromagnetic PFN powders by liquid phase synthesis and sintered ceramic samples by hot pressing method. The work show the influence of the synthesizing sol-gel method used on the electrophysical properties of PFN ceramic samples.

In the paper the structural, microstructural and dielectric properties of the PFN samples were investigated.

\section{Experiment}

\subsection{The first technology of the PFN material}

In the first chemical-wet sol-gel technology the $\mathrm{PbFe}_{1-x} \mathrm{Nb}_{x} \mathrm{O}_{3}$ powder was obtained using as precursors lead (II) acetate trihydrate $\mathrm{Pb}\left(\mathrm{CH}_{3} \mathrm{COO}\right)_{2} \cdot 3 \mathrm{H}_{2} \mathrm{O}(\mathrm{POCH})$, iron (III) nitrate nonahydrate $\mathrm{Fe}\left(\mathrm{NO}_{3}\right)_{3} \cdot 3 \mathrm{H}_{2} \mathrm{O}(\mathrm{POCH})$, niobium (V) ethoxide $\mathrm{Nb}\left(\mathrm{OC}_{2} \mathrm{H}_{5}\right)_{5}$ (Aldrich). In the process 2-metoxyethylate was a solvent and acetylacetone was a stabilizer (used both to control a hydrolysis of alcoholates and to protect against too quick gelation of the solution) while distilled water was used for the hydrolysis reaction. The lead acetate was solubilized in the 2-methoxyethylate by mixing and heating the solution. The iron nitride and the niobium ethylate were solubilized in 2-methoxyethylate, too. All components were weighed in a stoichiometric way (the

\footnotetext{
$\overline{\text { a przemyslaw.niemiec@us.edu.pl }}$
} 
lead (II) acetate trihydrate was added in excess because of its low evaporation temperature). The next step of the solgel technology was to mix obtained earlier solutions with their simultaneous heating. Afterwards the acetylacetone was added to the solution as a stabilizer as well as distilled water was added to start a hydrolysis process (a sol is formed). In order to hasten gelation process and to change the gel into a powder the solution was annealed under the IR lamp. In detail, the technological process was presented at work [21].

The PFN powder obtained after drying was mixed and to remove organic parts the calcined process was carried out. The process of remove organic parts $\left(T=600^{\circ} \mathrm{C}\right.$ for $t=2 \mathrm{~h}$ ) proceeds in a turbulent manner (gases are released from a powder in the form of brown smoke). The gelation process of the PFN material is shown in Fig. 1. The PFN ceramic samples obtained in first technology from iron (III) nitrate as PFN-n were marked.
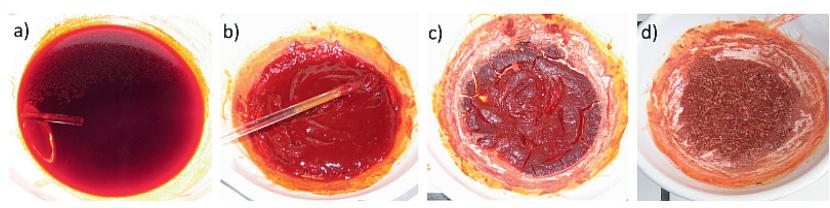

Figure 1. The gelation process of the PFN material in first solgel technology (PFN-n); a) solution of the PFN, b) the gelation process, c) the gelation process supported by IR rays, d) PFN-n powder.

\subsection{The second technology of the PFN material}

In the second sol-gel technology the $\mathrm{PbFe}_{1-x} \mathrm{Nb}_{x} \mathrm{O}_{3}$ powder was obtained using as precursors lead (II) acetate $\mathrm{Pb}\left(\mathrm{CH}_{3} \mathrm{COO}\right)_{2} \cdot 3 \mathrm{H}_{2} \mathrm{O}$ (POCH), niobium (V) ethoxide $\mathrm{Nb}\left(\mathrm{OC}_{2} \mathrm{H}_{5}\right)_{5}$ (Aldrich) and iron (III) oxalate $\mathrm{FeC}_{2} \mathrm{O}_{4}$ $\cdot 3 \mathrm{H}_{2} \mathrm{O}$ (Aldrich). The lead (II) acetate $\mathrm{Pb}\left(\mathrm{CH}_{3} \mathrm{COO}\right)_{2}$. $3 \mathrm{H}_{2} \mathrm{O}$ was dissolved in the ethyl glycol heating and mixing. The niobium ethylate $\mathrm{Nb}\left(\mathrm{OC}_{2} \mathrm{H}_{5}\right)_{5}$ was prepared in a similar way. In the case of the iron (III) oxalate a small amount of the ethyl glycol (POCH) and a small amount of a nitric acid was added and mixture was heated intensively. It results in a transition of the iron in the compound from II to III oxidation degree. In the next step, the three obtained component were mixed together and heating. After this, the acetylacetone was added (continuously heating) and the distilled water to start a hydrolysis process. In order to accelerate sol and gelation processes IR rays were used. In detail, the technological process was presented at work [22]. The gelation process of the PFN material is shown in Fig. 2.
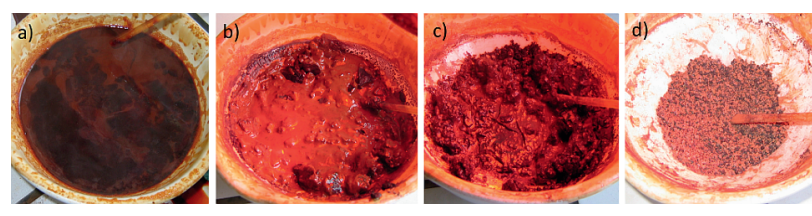

Figure 2. The gelation process of the PFN material in second sol-gel technology (PFN-ox); a) solution of the PFN, b-c) the gelation process supported by IR rays, d) PFN-ox powder.

After drying the PFN powder was mixed and next removes organic parts was done by calcinated method at the following conditions $600^{\circ} \mathrm{C} / 2 \mathrm{~h}$. The PFN ceramic samples obtained in second technology from iron (III) oxalate as PFN-ox were marked.

\subsection{The third technology of the PFN material}

In the third chemical-wet technology (solution precipitation method) the $\mathrm{PbFe}_{1-x} \mathrm{Nb}_{x} \mathrm{O}_{3}$ powder was obtained using as precursors ferric citrate monohydrate $\mathrm{C}_{6} \mathrm{H}_{5} \mathrm{FeO}_{7} \cdot \mathrm{H}_{2} \mathrm{O}$ (Fluka), lead (II) acetate trihydrate $\mathrm{Pb}\left(\mathrm{CH}_{3} \mathrm{COO}\right)_{2} \cdot 3 \mathrm{H}_{2} \mathrm{O}(\mathrm{POCH})$ and niobium $(\mathrm{V})$ ethoxide $\mathrm{Nb}\left(\mathrm{OC}_{2} \mathrm{H}_{5}\right)_{5}$ (Aldrich). The solution precipitation method used in PFN technology is a variation of the sol-gel method. The $\mathrm{Pb}\left(\mathrm{CH}_{3} \mathrm{COO}\right)_{2} \cdot 3 \mathrm{H}_{2} \mathrm{O}$ was dissolved in the $\mathrm{CH}_{3} \mathrm{COOH}$ acetic acid and simultaneously to $\mathrm{Nb}\left(\mathrm{OC}_{2} \mathrm{H}_{5}\right)_{5}$ ethylene glycol (POCH) with 2-methoxyethanol (Fluka) was added. Subsequently, to the ferric citrate $\mathrm{C}_{6} \mathrm{H}_{5} \mathrm{FeO}_{7} \cdot \mathrm{H}_{2} \mathrm{O}$ the distilled water was added and then the solution was heated. After this, the acetic acid was added to increase effectiveness of dissolving and it was brought to boiling (still stirring intensively). In the next step, all the component solutions were mixed together and heated simultaneously (this initiates the precipitation process), and the acetylacetone was add. Subsequently, the distilled water was added for the hydrolysis process. The obtained liquid solution was dried under an IR lamp in order to vaporize the solvent and to obtain ceramic powder.

Similar to the previous methods, after drying the PFN powder was mixed and removes organic parts was done by calcination route at following conditions: $600^{\circ} \mathrm{C} / 3 \mathrm{~h}$. In detail, the technological process was presented at work [23]. The process obtaining of the PFN material is shown in Fig. 3 and the PFN ceramic samples obtained in third technology from ferric citrate as PFN-c were marked.
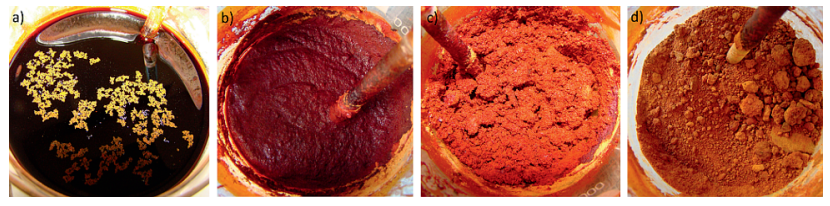

Figure 3. The co-precipitation method of the PFN material (PFN-c); a) the solution with precipitation process, b-c) the solvent vaporization supported by IR rays, d) PFN-c powder.

\subsection{Sintering of the PFN powders and samples preparation}

All the PFN powders obtained with three technologies after mixing process were pressed into the ceramic pellets. Densification was made by hot pressing method (HP) at following conditions: sintering temperature $T_{s}=1000^{\circ} \mathrm{C}$, holding time $t_{s}=1 \mathrm{~h}$ and the pressure $p_{s}=20$ $\mathrm{MPa}$.

The synthesized PFN powders were pressed into pellets with a thickness of $1.0 \mathrm{~mm}$. The mechanical stress was removed by annealing process at following condition $750^{\circ} \mathrm{C} / 0.25 \mathrm{~h}$, while for the electrical measurements the surfaces of the pellets were covered with the silver electrode $\left(850^{\circ} \mathrm{C} / 0.25 \mathrm{~h}\right)$. 


\subsection{Characterization}

The X-ray powder-diffraction data (XRD) of the sintered PFN samples were collected at room temperature $(R T)$ on a diffractometer Phillips X'Pert APD ( $\mathrm{Cu}-\mathrm{K} \alpha$ radiation). The data were collected in the $2 \theta$ range from $10^{\circ}$ to $65^{\circ}$ in steps of 0.02 degrees with an integration time of 4 s/step. Microstructure of the ceramic samples was observed by scanning electron microscopy JSM-7100F TTL LV. The stoichiometry of the PFN ceramics was studied with the EDS chemical composition analysis system. Prior to the SEM/EDS analyses the samples were coated with gold to provide electrical conductivity and to avoid any charging effects.

Dielectric measurements were carried out on a capacity bridge of a QuadTech 1920 Precision LCR Meter for a heating cycle (in temperature range from $20^{\circ} \mathrm{C}$ to $250^{\circ} \mathrm{C}$ ) and the measurements field frequency in the range from $100 \mathrm{~Hz}$ to $100 \mathrm{kHz}$.

\section{Results and discussion}

\subsection{X-ray diffraction analysis}

The X-ray powder-diffraction patterns at room temperature of PFN materials are presented in Fig. 4. The XRD measurements show that obtained materials have a perovskite structure and all samples are matching well with the tetragonal symmetry with $\mathrm{P} 4 \mathrm{~mm}$ space group (JCPDS card no. 04-009-5124). The XRD tests show that in the case of the PFN-c sample beside perovskite phase material has too, small amount of the pyrochlore phase $\left(\mathrm{Pb}_{2} \mathrm{Nb}_{2} \mathrm{O}_{7}\right)$. In case of the PFN-n sample there are very small amount foreign phase, while in the case of the PFN-ox there is no foreign (pyrochlore) phase.

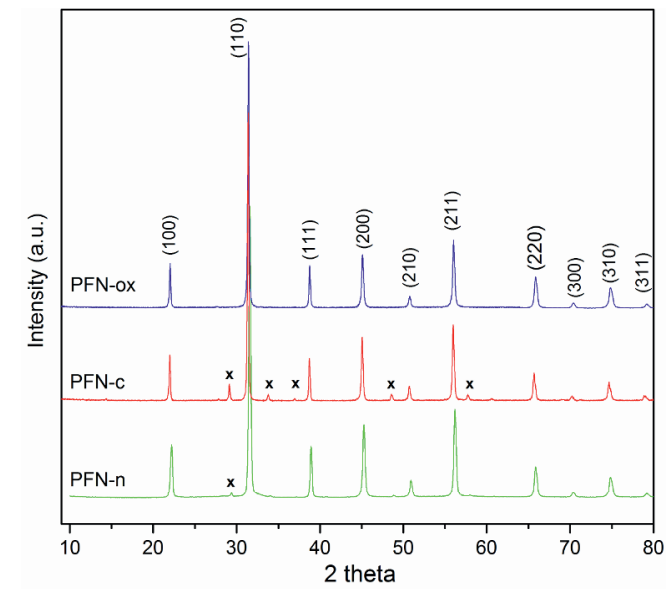

Figure 4. X-ray diffraction patterns of the PFN materials.

The percentage of perovskite phase in the PFN materials was calculated from the following equation (1):

$$
P_{\text {phase }}=\left[I_{\text {perov }} /\left(I_{\text {perov }}+I_{\text {pyroch }}\right)\right] \cdot 100 \%
$$

where: $I_{\text {perov }}$ is the intensity of highest perovskite peak (110) and $I_{\text {pyroch }}$ is the intensity of highest pyrochlore peak (222). Percentage of perovskite phase contents in case of the PFN-c sample is $94.4 \%$, whereas in case of the PFN-n sample is $97.86 \%$.

\subsection{Microstructural and EDS tests}

The images of morphology of fracture surfaces of the PFN samples obtained with different technologies are presented in Fig. 5. The microstructure of the PFN-n sample shows grain size heterogeneity. In the entire sample an angular of the ceramic grains is not fully correct.
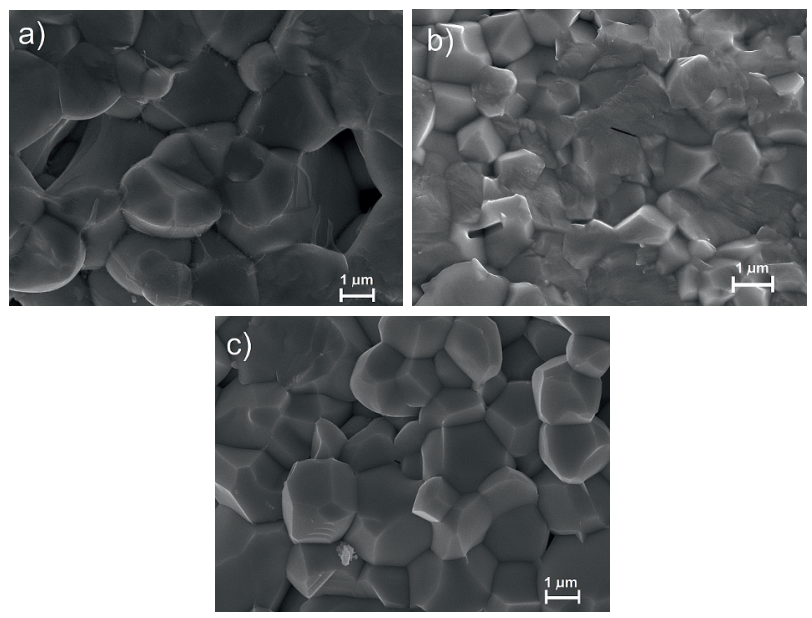

Figure 5. SEM images of the PFN ceramics: a) PFN-n, b) PFN-ox, c) PFN-c.

The breakthrough of the PFN-n sample is mixed and predominantly occurs through grain boundaries. However, when the fracture occurs through the grain, the surface morphology is solid, without any small elements originating from the cracked grain. This behavior indicates high mechanical strength of the inside of the grains and confirms the properly conducted technological process. The PFN-ox sample is characterized by a finegrained microstructure and densely packed, the grains of which have regular edges. The breakthrough of the PFNox sample occurs predominantly through the grain, which breaks in a compact solid manner (grain with a strongly consolidated structure does not break down into small elements). The PFN-ox ceramics have a fine closed porosity.

Among the ceramic samples obtained, the PFN-c one has the best microstructure. The grains are properly crystallized, and the microstructure shows high grain homogeneity. As in previous cases, there are closed pores in the volume of sample. The numerous grain agglomerates formed during the technology promote the formation of closed porosity. The sample breakthrough occurs mainly through grain boundaries, which is confirmed by properly selected technological sintering conditions of the ceramic ensuring high mechanical strength of the grain interior.

The EDS analysis (Fig. 6) confirmed the presence of constituent elements in the test PFN ceramic samples without the presence of foreign elements. In the Table 1 the percentage of the individual components of the PFN ceramics were given. Theoretical calculations were made 
on the basis of the chemical formulas of the obtained materials. In the case of the PFN-n sample, a small amount of iron and niobium deficiency and excess of lead are observed compared to theoretical calculations. In the case of the PFN-ox sample, a small amount of niobium deficiency and excess of iron has been demonstrated. In the case of the PFN-c sample lead and niobium deficiency and excess of iron are observed. For this technology percentage deviation from the theoretical composition are the largest.
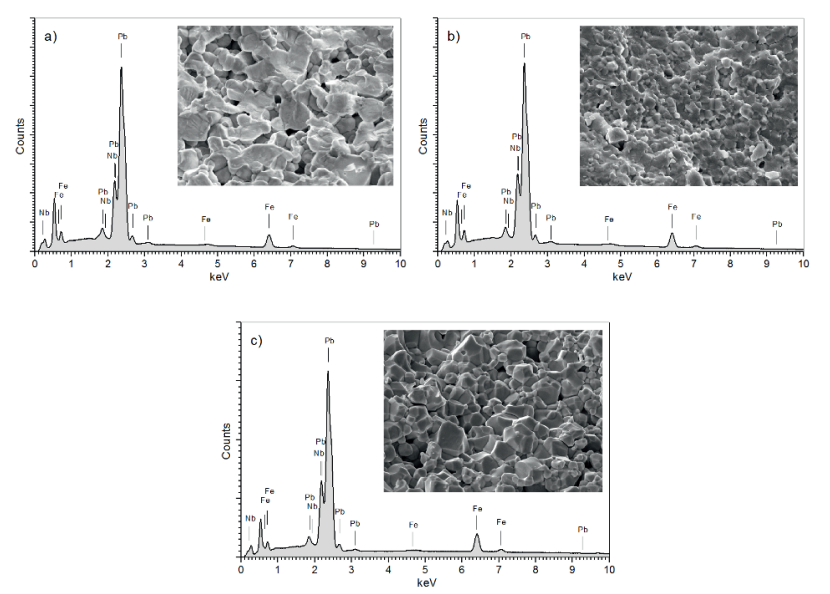

Figure 6. EDS testes of the PFN samples: a) PFN-n, b) PFN-ox, c) PFN-c.

Table 1. Theoretical and experimental percentages of elements of the PFN samples.

\begin{tabular}{|c|c|c|c|c|}
\hline & PFN & PFN-n & PFN-ox & PFN-c \\
\cline { 2 - 5 } & Theor. (\%) & Exptl. (\%) & Exptl. (\%) & Exptl. (\%) \\
\hline $\mathrm{PbO}$ & 67.72 & 69.34 & 67.84 & 65.34 \\
\hline $\mathrm{Fe}_{2} \mathrm{O}_{3}$ & 12.11 & 11.98 & 12.89 & 15.96 \\
\hline $\mathrm{Nb}_{2} \mathrm{O}_{5}$ & 20.16 & 18.68 & 19.27 & 18.71 \\
\hline
\end{tabular}

\subsection{Dielectric properties}

For the PFN ceramics, the maxima of dielectric permittivity (Fig. 7) are not as "sharp" as in the case of the ferroelectric single crystals or multicomponent PZTtype ceramics in which the phase transition occurs at a strictly defined temperature (Curie temperature) [24-25].

At room temperature $(R T)$, the PFN-n ceramics have high values of dielectric permittivity (for $1 \mathrm{kHz} \varepsilon=2,700$ ). On the temperature dependencies $\varepsilon(T)$ the graphs for the lower frequencies, do not show clear maximum dielectric permittivity occurring at the phase transition temperature. At higher frequencies (over $2 \mathrm{kHz}$ ), the characteristic maximum becomes clearer with broad form.

Of the samples tested, the PFN-ox ceramics have the highest values of dielectric permittivity and show a broad phase transition occurring over a wider range of temperatures. On the temperature dependencies $\varepsilon(T)$ with increasing frequency, frequency dispersion and a sudden drop in the dielectric permittivity are observed. For $1 \mathrm{kHz}$, the permittivity value maximum $\left(\varepsilon_{\max }\right)$ is 26,840 (at $T_{m}$ ) and approx. 2,600 (at $R T$ ). In comparison with the PFN ceramics obtained by free sintering method (at following conditions: $T_{s}=1100^{\circ} \mathrm{C}$ and $T_{s}=1150^{\circ} \mathrm{C}$ ) presented in the work [22], the PFN-ox ceramics have slightly lower $\varepsilon_{\max }$ values.
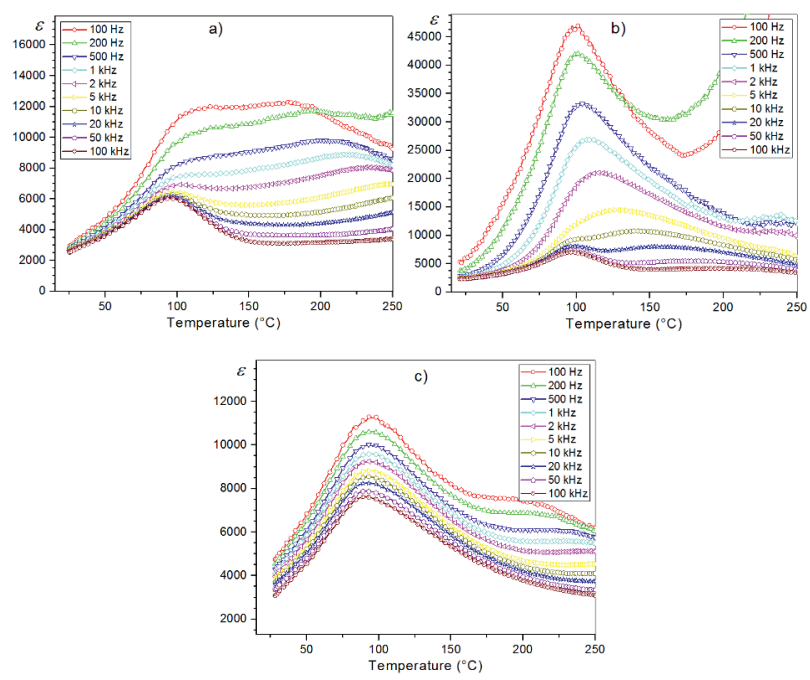

Figure 7. Temperature dependencies of dielectric permittivity (ع) for the PFN ceramics: a) PFN-n, b) PFN-ox, c) PFN-c.

The PFN-c ceramics have a broad phase transition but occurring within a narrow temperature range. There is no frequency dispersion with the frequency increase (the characteristic maximum phase transition occurs at the same temperature). From the tested PFN samples, the values of dielectric permittivity at $R T$ are the highest. For $1 \mathrm{kHz}$, the value of the dielectric permittivity maximum (at $T_{C}$ ) is 9,590 and at $R T \varepsilon=4,100$. Compared with the PFN ceramics obtained by FS [23], the PFN-c ceramics have a lesser degree of blurring the phase transition and higher values of $\varepsilon_{\max }$. In the work [23] the value of $\varepsilon_{\max }$ is 5,110 .

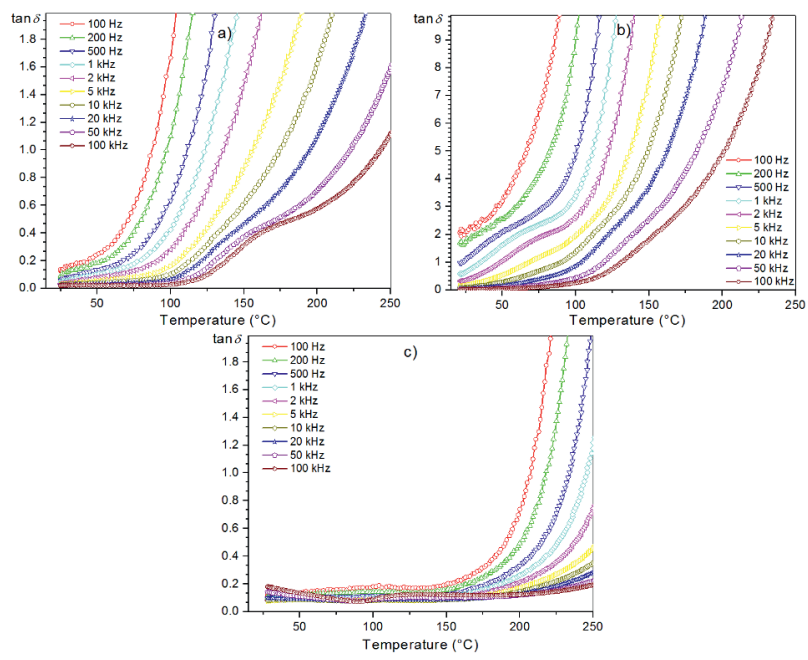

Figure 8. Temperature dependencies of dielectric loss tangent $(\tan \delta$ ) for the PFN ceramics: a) PFN-n, b) PFN-ox, c) PFN-c.

Fig. 8 presents the results of temperature measurements of dielectric loss for PFN ceramic samples. 
In the case of the PFN-n ceramics with increasing temperature, dielectric loss increase. Just before the phase transition temperature, the values of the $\tan \delta$ increase rapidly. At $R T(1 \mathrm{kHz})$, value of the dielectric loss is 0.040. In comparison with the PFN ceramics obtained by $\mathrm{HP}$ at higher temperatures $\left(T_{s}=1050^{\circ} \mathrm{C}\right.$ and $\left.T_{s}=1100^{\circ} \mathrm{C}\right)$ presented in the work [21], the PFN-n ceramics have lower value of dielectric loss.

Comparing the obtained PFN samples, the PFN-ox ceramics have the highest dielectric loss. The high values of the $\tan \delta$ occur both at low temperatures $(0.58$ for 1 $\mathrm{kHz}$ ) as well as at higher temperatures, where the increase in dielectric loss is very high. In comparison with the PFN ceramics obtained by FS method [22], the PFN-ox ceramics show slightly higher dielectric loss.

The PFN-c ceramics exhibits the lowest dielectric loss over the entire temperature and frequency measurement area. At $R T(1 \mathrm{kHz})$, dielectric loss is 0.086 , while at $T_{m}$, 0.099 . Low dielectric loss may be associated with the presence of more pyrochlore phase (as shown by XRD tests), which partially reduces the electrical conductivity from the intergranular areas, reducing values of dielectric loss. In comparison with the PFN ceramics obtained by FS method [23], the PFN-n ceramics have significantly lower dielectric loss.

\section{Conclusion}

The paper shows the technological process of the three kinds of chemical-wet method synthesis of the multiferroic $\mathrm{PbFe}_{1 / 2} \mathrm{Nb}_{1 / 2} \mathrm{O}_{3}$ materials and sintered by hot pressing method. Differences between individual technologies were related to the change in Fe component, i.e. iron nitrate obtained by first sol-gel technology (PFN$\mathrm{n})$, iron oxalate obtained by second sol-gel technology (PFN-ox) and iron citrate obtained by third technology (PFN-c).

The XRD measurements show that despite the pyrochlore phase, the PFN-c ceramics have the best dielectric properties, i.e. the optimum of the dielectric permittivity and low dielectric loss, compare to the all obtained PFN ceramic samples. SEM microstructure tests of the PFN ceramic samples confirm the properly conducted technological processes.

Comparing three technologies of the PFN syntheses performed by the sol-gel method, it can be concluded that the optimal technology is the process using iron citrate (third method). However, this process requires elimination or minimization of a pyrochlore phase in the PFN ceramics as well as reduction of the porosity ceramic samples (eliminating creation of hard clusters agglomerate of the powder that promote the formation of significant closed porosity). Minimizing the possibility of powder agglomeration formation can be partially eliminated by breaking them with ultrasound methods. The results of such research will be the subject of the next work of the authors.

\section{Acknowledgements}

The authors thank the Dr Katarzyna Osińska for help in the technological process.

\section{References}

1. D. Khomskii, Physics 2, 20 (2009).

2. H. Schmid, J. Phys.: Condens. Matter 20, 434201 (2008)

3. K.F. Wang, J.-M. Liu, Z.F. Ren, Adv. Phys. 58, 321 (2009)

4. M.H. Lente, J.D.S. Guerra, G.K.S. de Souza, B.M. Fraygola, C.F.V. Raigoza, D. Garcia, J.A. Eiras, Phys. Rev. B 78, 054109 (2008)

5. D. Bochenek, Z. Surowiak, J. Krok-Kowalski, J. Poltierova-Vejpravova, J. Electroceram. 25, 122 (2010)

6. J.F. Scott, J. Mater. Chem. 22, 4567 (2012)

7. Y. Xu, Ferroelectric Materials and Their Applications. (Elsevier, North Holland, New York, 1991)

8. D. Bochenek, J. Dudek, Eur. Phys. J.-Spec. Top. 154, 19 (2008)

9. D. Bochenek, P. Niemiec, P. Guzdek, M. Wzorek, Mater. Chem. Phys. 195, 199 (2017)

10. X. Meng, K.Z. Baba-Kishi, G.K.H. Pang, H.L. Chan, CH.-L. Choy, H. Luo, Ferroelectrics 297, 57 (2003).

11. K. Tahmasebi, A. Barzegar, Transaction F: Nanotechnology 17, 108 (2010)

12. D. Bochenek, P. Niemiec, R. Skulski, A. Chrobak, P. Wawrzała, Mater. Chem. Phys. 157, 116 (2015).

13. S. Srinivas, J.Y. Li, Acta Mater. 53, 4135-4142 (2005)

14. J. Kulawik, D. Szwagierczak, P. Guzdek, J. Magn. Magn. Mater. 324, 3052 (2012)

15. L.M. Hrib, O.F. Caltun, J. Alloys Compds. 509, 6644 (2011)

16. O. Raymond, R. Font, N. Suárez-Almodovar, J. Portelles, J. M. Siqueiros, J. Appl. Phys. 97, 084107 (2005)

17. Y.-Ch. Liou, Ch.-Yu Shih, Ch.-H. Yu, Jpn. J. Appl. Phys. 41, 3829 (2002)

18. S. Ananta, N.W. Thomas, J. Eur. Ceram. Soc. 19, 2917 (1999)

19. S.B. Majumder, S. Bhattacharyya, R.S. Katiyar, A. Manivannan, P. Dutta, M.S. Seehra, J. Appl. Phys. 99, 024108 (2006)

20. X. Gao, J. Xue, J. Wang, T. Yu, Z.X. Shen, J. Am. Ceram. Soc. 85, 565 (2002).

21. D. Bochenek, Z. Surowiak, J. Alloy. Compd. 480, 732 (2009)

22. D. Bochenek, R. Zachariasz, Materialwiss. Werkst. 46, 294 (2015)

23. D. Bochenek, J. Alloy. Compd. 504, 508 (2010)

24. L.B. Kong, J. Ma, H.T. Huang, W. Zhu, O.K. Tan, Mater. Lett. 50, 129 (2001)

25. B. Wodecka-Duś, D. Czekaj, Arch. Metall. Mater. 54 923 (2009) 